\title{
Photodegradation of organic compounds in water
}

\author{
Krzysztof Brodzik, Jerzy Walendziewski, Marek Stolarski \\ Wroctaw University of Technology, Faculty of Chemistry, Department of Fuels Chemistry and Technology, \\ ul. Gdańska 7/9, 50-344 Wrocław, Poland, e-mail: jerzy.walendziewski@pwrwroc.pl
}

\begin{abstract}
The application of photocatalytic processes for the decontamination treatment of polluted water has inspired very extensive studies. Titanium dioxide with its large band gap energy and appropriate redox potential was found as one of the most promising semiconductors for the photodegradation of pollutants in the water as well as in gas phase. The titania-silica aerogels obtained by a simple co-hydrolysis method was applied in the photodegradation of the model organic compound. Different ageing times and heat treatment temperatures were found to influence both the activity and the textural properties of the photocatalysts. The obtained aerogels are efficient photodegradation catalysts of methylene blue and allow a removal up to 98 and $78 \%$ of the model pollutant from 20 and $500 \mathrm{ppm}$ solutions, respectively.
\end{abstract}

Keywords: Photodegradation, photocatalyst, titania, silica titania, aerogels.

Presented at VII Conference Wasteless Technologies and Waste Management in Chemical Industry and Agriculture, Międzyzdroje, 12 - 15 June, 2007.

\section{INTRODUCTION}

Application of heterogeneous photocatalysis, one of Advanced Oxidation Processes (AOP), have gained much attention in recent years. The main advantage of the photodegradation of pollutants in water is their total mineralization to simple, non-toxic products e.g. $\mathrm{CO}_{2}$ and water. The process involves photoexcitation of the semiconductor photocatalyst allowing the generation of highly reactive electron-hole pairs. Titanium dioxide, mainly in its anatase crystalline form, with its large band gap energy, non-toxic character, photo stability and biological and chemical inertness was found as an almost ideal photocatalyst but cannot be induced via the visible light ${ }^{1}$. The efforts for the improvement of the photocatalytic activity of titania can be divided into two main groups, concerning: (i) shifting the absorption band gap to the visible light portion of the spectra and (ii) enhancing the $\mathrm{TiO}_{2}$ photoactivity in the near $\mathrm{UV}^{2}$.

Recently the titania-silica systems in the form of aerogels have been suggested to be more active than the pure titania catalysts. In those aerogels silica provides an excellent porous structure with an extremely high surfaced area and porosity, allowing good adsorption of pollutants. In addition, it is transparent for irradiation in the range needed for the photo excitation of the titania species which can be highly dispersed in/on the silica matrix. The preparation procedure of aerogel is based on the sol-gel process and involves hydrolysis of the metal precursors followed by a condensation of the obtained species with the formation of gel. In order to obtain aerogels from wet gels, the applied solvent must be replaced for air. To prevent the collapse of a highly-porous gel structure during the removal of the solvent, special drying procedures are applied, i.e. low or high temperature drying. The preparation process allows obtaining the highly-dispersed titania species which can be better utilized in the photocatalytic process. The titania-silica aerogel were effectively applied in the photodegradation of benzene ${ }^{3}$, cyanides ${ }^{4,5}$, phenol $^{6}$ and its para-derivatives ${ }^{7}$ and others. Our recent study on the photodegradation of salicylic acid ${ }^{8}$ also indicates bet- ter utilization of titania particles in the titania-silica aerogels than in the case of pure $\mathrm{TiO}_{2}$ catalysts.

The objective of this study is to correlate the influence of titania content, the heat treatment temperature and the ageing time of the titania-silica aerogels on the efficiency of the photodegradation process of polluted waters. The aerogels were prepared using the co-hydrolysis of titania and silica precursors and methylene blue (MB) was chosen as a model organic compound.

\section{EXPERIMENTAL}

The aerogels were obtained using the method described previously $^{\mathbf{8}}$. Tetraethyl orthosilicate (TEOS, Fluka) and tetrabutyl orthotitanate (TBT, Aldrich) were selected as the aerogel precursors. Two anhydrous alcohols: methanol (POCh S.A.; critical temperature, $\mathrm{T}_{\mathrm{K}}=240^{\circ} \mathrm{C}$; critical pressure, $\mathrm{p}_{\mathrm{K}}=78.7 \mathrm{~atm}$ ) and ethanol (POCh S.A.; $\left.\mathrm{T}_{\mathrm{K}}=243^{\circ} \mathrm{C}, \mathrm{p}_{\mathrm{K}}=63.7 \mathrm{~atm}\right)$ were used as the reaction mixture solvents. All the syntheses were carried at room temperature. The method was based on mixing both precursors, followed by an addition of the deionized water, ethanol and the hydrolysis catalyst hydrochloric acid with the molar ratio Si: $\mathrm{H}_{2} \mathrm{O}: \mathrm{EtOH}: \mathrm{HCl}=1: 4: 25.6: 0.022$. The amount of titania precursor was set to obtain 10,20 and $30 \mathrm{wt} \% \mathrm{TiO}_{2}$ in the resulting aerogel. After $15 \mathrm{~min}$ of mixing the reagent, additional amount of water, methanol (Ti: $\mathrm{H}_{2} \mathrm{O}: \mathrm{MeOH}$ molar ratio was 1:4:25) and ammonia water were introduced so as to bring the system $\mathrm{pH}$ to the neutral value and induce its condensation. The obtained sols were left for gelation and ageing for $18 \mathrm{~h}$ and 7 or 14 days. Drying of the obtained gels was realized in a reactor at $280^{\circ} \mathrm{C}$ and $11 \mathrm{MPa}$, under the conditions called as high temperature supercritical drying (HTSCD). The precise drying procedure was described elsewhere [8]. The name of the samples corresponds to their preparation procedure, for example, in the sample name 20a 7, „20" corresponds to $20 \mathrm{wt} \%$ of $\mathrm{TiO}_{2}$ and ,a 7 " to the ageing period, in this case 7 days.

The prepared aerogels were calcined at 500 and $600^{\circ} \mathrm{C}$ for $4 \mathrm{~h}$ in air atmosphere with the heating rate $100^{\circ} \mathrm{C} / \mathrm{h}$. The textural characteristics i.e. Brunauer-Emmett-Teller 
(BET) surface area, volumes of micro- (de Boer model) and mesopores, pore size distributions (Barrett-JoynerHalenda, BJH model) and average pore radius based on the BET surface area, were determined by the $\mathrm{N}_{2}$ adsorption/desorption method (Quantachrome Autosorb 1C). The crystal form of $\mathrm{TiO}_{2}$ was investigated by the XRD analysis (Philips X'Pert system, detector: type PW 1711 and source: $\mathrm{Cu} \mathrm{K} \alpha$, Ni filter, $40 \mathrm{kV}, 30 \mathrm{~mA}$ ). The presence of the Ti-O-Si bonding was studied with the FTIR (Thermo Electron Nexus FTIR).

The photocatalytic tests were carried out by using 400 $\mathrm{cm}^{3}$ of 20 or $50 \mathrm{ppm} \mathrm{MB}$ solutions in water and $0.2 \mathrm{~g}$ of the catalyst in the photoreactor with the medium pressure mercury lamp (ACE GLASS Inc.). Each time the system containing the ground aerogel and model pollutant solution was mixed for $30 \mathrm{~min}$ with the magnetic stirrer in darkness so as to allow establishing of the adsorption equilibrium. The photocatalytic activity of the titaniasilica aerogels was evaluated by determination of the methylene blue decomposition level i.e. lowering its concentration in the water solution, using the UV-Vis spectrometer (Hitachi U-2001).

\section{RESULTS}

The textural properties of the obtained titania-silica aerogels are summarized in Table 1 . The increase of titania content in the catalysts aged for $18 \mathrm{~h}$ leads to decrease both the BET surface areas and the mesopores volumes. While the surface area is taken into account, differences between the aerogels decay while the aging time elongates. This effect can be attributed to the fact that after $18 \mathrm{~h}$ of ageing the whole system is far from equilibrium, some hydrolyzed species are still present in the solvent which fills the pore structure and may undergo further condensations with the already formed gel matrix. The alcoholysis process, competitive to the hydrolysis reaction, may also influence the gel structure. During the ageing of gels, condensation of the surface $\mathrm{Si}-\mathrm{OH}$ and $\mathrm{Ti}-\mathrm{OH}$ groups occurs, leading to the reconstruction of the gel matrix and diminishing of pore diameters, as it can be seen in Fig. 1 presenting the $\mathrm{BJH}$ pore size distributions. Narrowing of pores is clearly visible in the case of 10 and $20 \mathrm{wt} \% \mathrm{TiO}_{2}$ aerogels, while $30 \mathrm{wt} \%$ one indicates rather pores broadening. Different behavior of the catalysts may be attributed to the changes in the condensation process occurring during the ageing period.

The XRD analysis of the etitania-silica aerogels proved that only one crystalline form is generated during the heat treatment at 500 and $600{ }^{\circ} \mathrm{C}$, namely it is the anatase form of titania. An attempt was made to determine the amount of crystalline phase in the prepared samples. It was done using silicon as the internal standard (Fig. 2). In the case of 10a1 and 30a1 aerogels the amount of anatase decreases depending on the calcination temperature, from 9.3 to 9.0 and from 27.4 to $25.8 \%$, respectively. These measurements also indicate that almost all titania in the prepared aerogels forms anatase crystals. In the case of the 20a1 sample an increase in the anatase amount was noticed, from 15.3 to $16.0 \%$, it is worth noticing that in this case a relatively large amount of titania remains amorphous. Besides the crystalline form of titania, the FTIR spectra reveal the presence of the Ti-O-Si bonding in the all prepared photocatalysts (Fig. 3). Those data allow us to state that the studied titania-silica aerogels are the mixtures of titania on silica as well as the titania-silica mixed oxides.

The experimental data obtained from the photocatalytic tests are summarized in Table 2. As it was pointed out before starting the photocatalytic test, each photodegradation system was kept for 30 min in darkness under vigorous stirring. The samples obtained just before turning on the UV lamp served as the indicators of the amount of adsorbed MB. The amount of the adsorbed

Table 1. The influence of the titania content and the ageing time on the textural properties of the titania-silica aerogels

\begin{tabular}{|c|c|c|c|c|c|}
\hline Aerogel & Ageing & BET, $\mathrm{m}^{2} / \mathrm{g}$ & $\mathrm{V}_{\text {micro }}, \mathrm{cm}^{3} / \mathrm{g}$ & $\mathrm{V}_{\text {meso }}, \mathrm{cm}^{3} / \mathrm{g}$ & APD, $n m$ \\
\hline $10 a 1$ & \multirow{3}{*}{$18 \mathrm{~h}$} & 1031 & 0.0044 & 1.895 & 20.8 \\
\hline $20 a 1$ & & 875 & 0.0264 & 1.679 & 25.6 \\
\hline $30 a 1$ & & 592 & 0.0078 & 1.104 & 17.7 \\
\hline $20 a 7$ & \multirow[t]{2}{*}{$7 d$} & 768 & 0.0060 & 1.356 & 17.6 \\
\hline $30 a 7$ & & 670 & 0.0016 & 1.420 & 19.5 \\
\hline 10a14 & \multirow{3}{*}{$14 d$} & 896 & 0.0026 & 1.878 & 19.6 \\
\hline $20 a 14$ & & 824 & 0.0046 & 1.756 & 18.1 \\
\hline $30 a 14$ & & 695 & 0.0116 & 1.409 & 17.3 \\
\hline
\end{tabular}

$\left(\mathrm{V}_{\text {micro }}\right.$ - micropores volume, $\mathrm{V}_{\text {meso }}$ - mesopores volume, $\mathrm{APD}$ - average pore diameter)

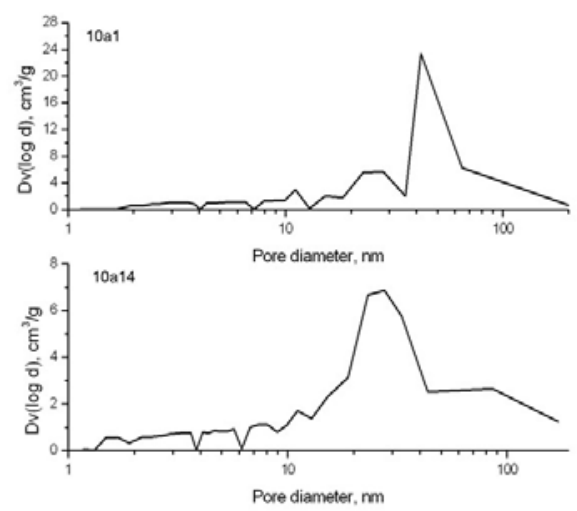

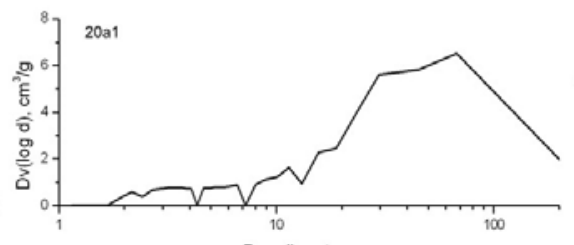
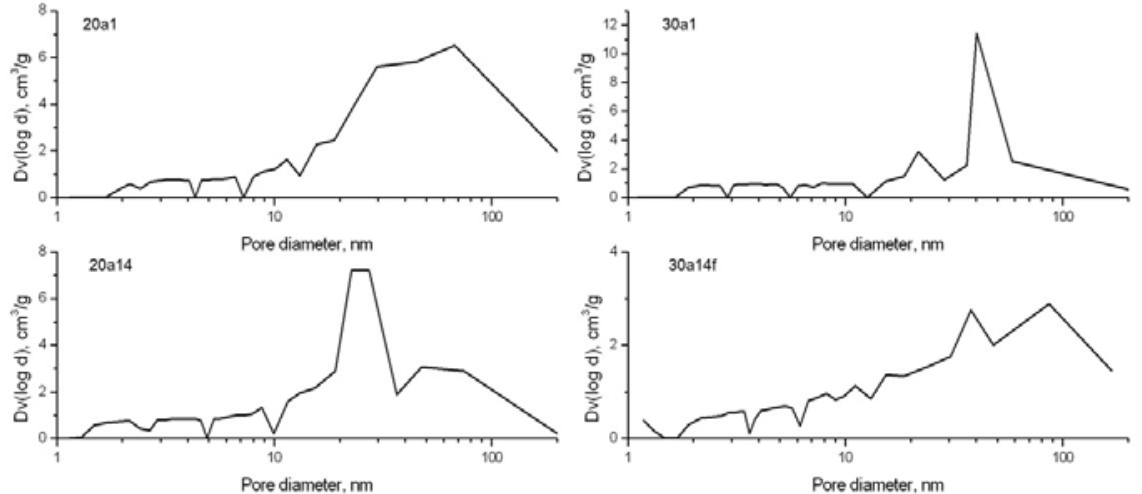

Figure 1. BJH pore size distributions determined for the $18 \mathrm{~h}$ or 14 days aged aerogels 
Table 2. The photocatalytic efficiency of the titania-silica aerogels

\begin{tabular}{|c|c|c|c|c|}
\hline Aerogel & Ageing & Heat treatment & Adsorption, \% * & Conversion, $\%$ \\
\hline $10 \mathrm{a} 1$ & \multirow{6}{*}{$18 \mathrm{~h}$} & $500^{\circ} \mathrm{C}$ & $52.6 / 32.0$ & $87.2 / 45.4$ \\
\hline 10a1 & & $600^{\circ} \mathrm{C}$ & $66.5 /-$ & $96.5 /-$ \\
\hline $20 \mathrm{a} 1$ & & $500^{\circ} \mathrm{C}$ & $52.1 / 36.6$ & $93.6 / 65.8$ \\
\hline $20 a 1$ & & $600^{\circ} \mathrm{C}$ & $72.0 /-$ & $97.5 /-$ \\
\hline $30 a 1$ & & $500^{\circ} \mathrm{C}$ & $41.5 / 42.7$ & $96.5 / 76.2$ \\
\hline $30 a 1$ & & $600^{\circ} \mathrm{C}$ & $74.5 /-$ & $95.0 /-$ \\
\hline $10 a 7$ & $7 d$ & \multirow{6}{*}{$500^{\circ} \mathrm{C}$} & $-/ 45.3$ & $-/ 52.0$ \\
\hline $10 a 14$ & $14 d$ & & $-/ 30.1$ & $-/ 59.8$ \\
\hline $20 a 7$ & $7 d$ & & $-/ 31.7$ & $-/ 62.7$ \\
\hline $20 a 14$ & $14 d$ & & $-/ 32.2$ & $-/ 68.8$ \\
\hline $30 \mathrm{a} 7$ & $7 d$ & & $-/ 37.7$ & -168.8 \\
\hline $30 a 14$ & $14 d$ & & $-/ 30.4$ & $-/ 77.6$ \\
\hline
\end{tabular}

( ${ }^{*}$ - the values represent the data obtained from the $20 / 50 \mathrm{ppm}$ solutions)
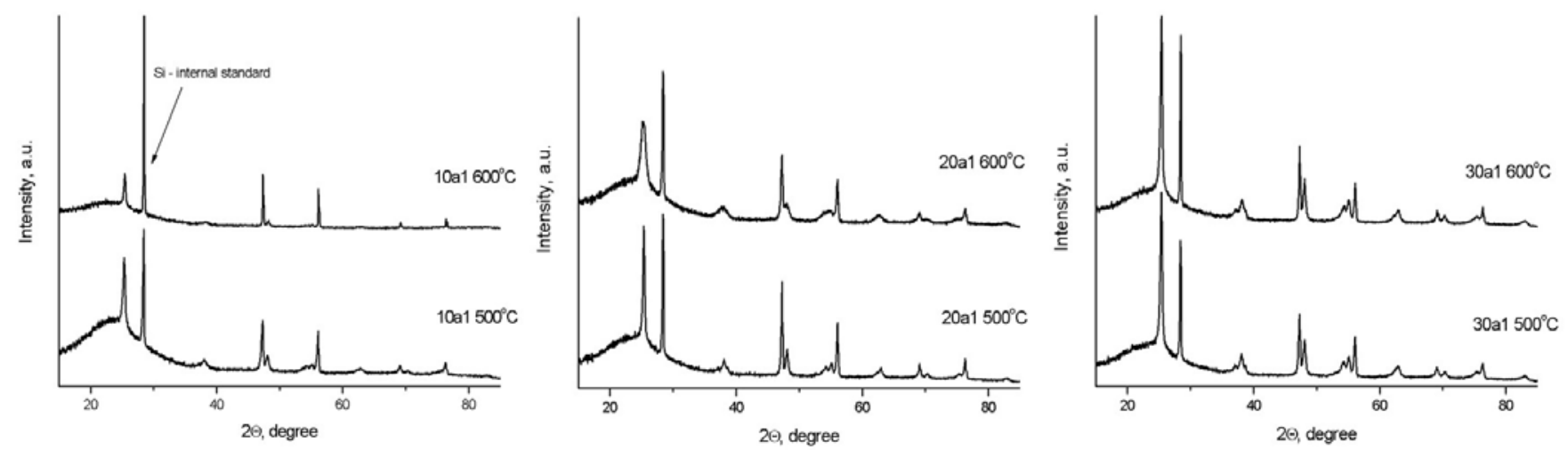

Figure 2. XRD patterns of the $18 \mathrm{~h}$ aged aerogel samples

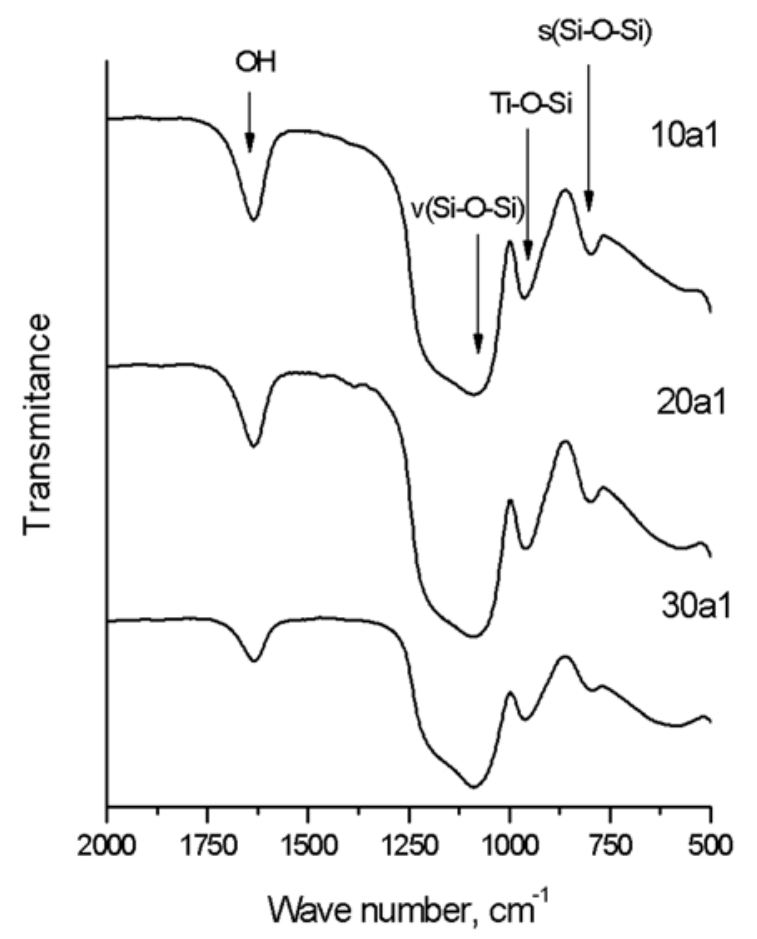

Figure 3.

pollutant correlates with the efficiency of its removal during irradiation. In the case of the first part of the study based on the application of aerogels subjected to different thermal treatment, $20 \mathrm{ppm} \mathrm{MB}$ containing solutions were used. The conversion increase in the case of the application of 10a1 samples can be straightly correlated only to the effect of the preadsorbed model organic compound because of the very similar anatase contents. The conversion decrease in the case of 30a1 catalyst with increasing the heat treatment temperature accompanied by the increase in the preadsorbed MB quantity, can be the consequence of the anatase content decrease. Despite the difference in the adsorption of the model pollutant from the 20 ppm solutions, all the titania-silica aerogels indicated high efficiency with the conversions up to $97.5 \%$.

The influence of the ageing time on the photocatalytic performance of the titania-silica aerogels was investigated using higher concentrations of methylene blue i.e. $50 \mathrm{ppm}$. Elongation of the ageing time from 18 hours to 14 days in the case of $10 \mathrm{wt} \%$ of $\mathrm{TiO}_{2}$ containing aerogels resulted in the heightening the conversion from 45.4 up to $59.8 \%$, but the highest amount of the preadsorbed MB was noticed in the case of the $10 \mathrm{a} 7$ sample. The adsorption performance of the photocatalysts with a higher titania content can be connected with their average pore diameters, i.e. diminishing the pores diameters lead to the decrease in the amount of the preadsorbed organic compound. Only in the case of the 10a sample the photocatalytic efficiency raises with the elongation of the ageing time. The seven days ageing in the case of both, 20 and $30 \mathrm{wt} \% \mathrm{TiO}_{2}$ aerogels leads to a decrease of their activity in comparison to the $18 \mathrm{~h}$ aged samples, while 14 days ageing produces the aerogels of the highest activity. Concluding, it can be noted that the ageing time of the aerogels obtained by the co-hydrolysis of the titania and silica precursors can strongly enhance the photocatalytic activity of the aerogels with the low titania amount, presumably due to the increasing availability of active sites. The materials containing more titania however, need to be aged for longer time than a week, to attain activity they posses after short (18h) ageing. 


\section{CONCLUSIONS}

Highly porous titania-silica aerogels with the surface areas up to $1000 \mathrm{~m}^{2} / \mathrm{g}$ can be prepared using a simple cohydrolysis method. Thermal treatment plays major role in the preadsorption of pollutants from water solutions and may also enhance the photocatalysts' performance. Ageing time strongly influences the textural properties of aerogels but its effect on their photoactivity is strongly dependent on the titania content. Although the preadsorption of the pollutant may increase the process efficiency, great attention should be paid to the adsorption capacity so as a large amount of the pollutant does not mask the active sites and inhibit their photo excitation.

\section{ACKNOWLEDGMENTS}

This work was supported by The Polish Ministry of Scientific Research and Information Technology (Project no. 3 T09B 03 228).

\section{LITERATURE CITED}

(1) Bahtkhande D. S., Pangarkar V. G., Beenackers A. A. C. M.: Photocatalytic degradation for environmental applications - a review, J. Chem. Techno. Biotechnol., 2002, 77, 102.

(2) Carp O., Huisman C. L., Reller A.: Photoinduced reactivity of titanium dioxide, Prog. Solid State Chem., 2004, $32,33$.

(3) Yoda S., Ohtake K., Takebayashi Y., Sugeta T., Sako T., Sato T.: Preparation of titania-impregnated silica aerogels and their application to removal of benzene in air, J. Mater. Chem., 2000, 10, 2151.

(4) Ahmed M. S., Attia Y. A.: Aerogel materials for photocatalytic detoxification of cyanide wastes in water, J. Non Cryst. Solids, 1995, 186, 402.

(5) Ismail A. A., Ibrahim I. A., Ahmed M. S.: Mohamed R.M., El-Shall H., Sol-gel synthesis of titania-silica photocatalyst for cyanide photodegradation, J. Photochem. Photobiol. A, 2004, 163, 445.

(6) Deng Z., Wang J., Zhang Y., Weng Z., Zhang Z., Zhou B., Shen B., Cheng L.: Preparation and photocatalytic activity of $\mathrm{TiO}_{2}-\mathrm{SiO}_{2}$ binary aerogels, Nanostruct. Mater., 1999, 11, 1313.

(7) Malinowska B., Walendziewski J., Robert D., Weber J. V., Stolarski M.: The study of photocatalytic activities of titania and titania-silica aerogels, Appl. Cat. B, 2003, 46, 441.

(8) Brodzik K., Walendziewski J., Stolarski M., Van Ginneken L., Elst K., Meynen V.: The influence of preparation method on the physicochemical properties of titaniasilica aerogels, J. Porous Mater., in press (on-line first, DOI 10.1007/s10934-006-9027-9). 